\title{
Aplicativo iOS para coleta autônoma de imagens e monitoramento do meio agrícola
}

\author{
Gustavo César Visentini ${ }^{1}$, Willingthon Pavan $^{1}$, Carlos A. Holbig ${ }^{1}$, Jose Mauricio Fernades ${ }^{1,2}$ \\ ${ }^{1}$ Universidade de Passo Fundo (UPF) \\ Passo Fundo - RS - Brazil \\ 119692@upf.br, pavan@upf.br, holbig@upf.br \\ ${ }^{2}$ Embrapa Trigo \\ Passo Fundo - RS - Brazil \\ mauricio.fernandesembrapa.br
}

\begin{abstract}
Agricultural monitoring has relied on numerous methods and technologies, where the Drones' usage is one of the most current. This paper presents an application for mapping and control using DJI Drones, developed for the iOS platform. With this application it is possible to collect in a simplified and automated way images, what can be used to create mosaics, being able to assist in the most varied projects.
\end{abstract}

Resumo. O monitoramento agrícola tem contado com inúmeros métodos e tecnologias, sendo o uso de Drones um dos mais atuais. Neste trabalho apresentase um aplicativo para controle e mapeamento aéreo utilizando Drones DJI, desenvolvido para a plataforma iOS. Com o aplicativo pode-se coletar de forma simplificada e automatizada imagens para a criação de mosaicos, os quais podem auxiliar nos mais variados projetos.

\section{Introdução}

Atualmente os Drones tornaram-se aliados à Agricultura de Precisão permitindo detectar e monitorar grandes áreas em tempo real. Por meio do uso de imagens pode-se identificar, por exemplo, pragas e áreas com necessidade de adubação, buscando auxiliar na tomada de decisões, assim como na busca de um aumento de produtividade e redução de custos. Outro fator que agrega vantagens ao uso de Drones é a grande variedade de equipamentos disponíveis atualmente no mercado [Artioli and Beloni 2016].

Entretanto, a operação destes equipamentos demanda conhecimento e treinamento, tanto com o equipamento como com o software, os quais muitas vezes apresentam uma grande curva de aprendizado. Aplicativos como o DJI Ground Station PRO [DJI-GS-PRO 2018], podem ser utilizados para realizar tarefas como o mapeamento, porém, seu uso tornar-se difícil para usuários com pouca ou sem experiência na área.

Neste contexto, o aplicativo aqui apresentado busca oferecer uma interface simples e de fácil uso para os usuários do meio agrícola, abstraindo todo o trabalho de cálculos e configurações do Drone. 


\section{O Aplicativo Auto Capture e seu funcionamento}

$\mathrm{O}$ aplicativo desenvolvido, denominado Auto Capture, consiste em uma ferramenta mobile que visa simplificar o processo de coletas de imagens, eliminando o trabalho como cálculos e procedimentos que deveriam ser feitos na coleta de imagens sem o auxilio de um aplicativo. Se faz necessário, apenas, selecionar o local de voo por meio de waypoints $^{1}$ em um mapa e informar a altura de voo desejada.

Para a obtenção correta das imagens da área a ser monitorada, faz-se necessário manter uma sobreposição (overlapping) entre as imagens coletadas. Segundo Ferreira [Ferreira et al. 2013] para obter-se um bom resultado final no processamento das imagens deve-se respeitar uma sobreposição lateral (sidelap) de 60\% e uma sobreposição longitudinal (frontlap) de 70\% em média. Na Figura 1 observamos um caso de frontlap.

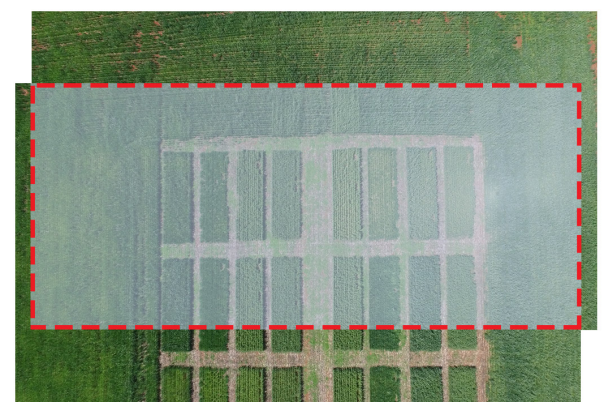

Figura 1. Sobreposição longitudinal entre imagens vizinhas denominada frontlap, sendo neste caso de aproximadamente $70 \%$.

O aplicativo foi projetado e desenvolvido para a plataforma iOS (compatível com iPhone/iPad e iPod Touch) [Henrique et al. ], utilizando a linguagem a Swift 4.0. Para o processo de comunicação entre o aplicativo e o Drone, foi utilizado o SDK (Software Development Kit) da DJI [DJI-Developer 2018], sendo que a versão do SDK utilizado foi a Mobile SDK. O SDK permite a criação de aplicativos para Android ou IOS, encapsulando a comunicação e utilizando de sensores e componentes do Drone, como o controle de voo autônomo, de mapa, além do controle de câmera para capturar imagens.

Para manter o overlapping a distância entre a captura das imagens deve ser respeitada, desta forma o cálculo é feito levando em consideração a altura, que é informada pelo usuário. Quanto maior for a distância entre uma imagem e outra aumenta, que por sua vez também aumentará a velocidade do voo. Levando em consideração estas variáveis, o aplicativo calcula o momento exato de captura de cada imagem. Como exemplo, após selecionar 50 metros de altura, o aplicativo calcula automaticamente que a velocidade de voo deve ser de $10 \mathrm{~m} / \mathrm{s}$ e a distância de captura de uma imagem para a outra será de 30 metros. Estes dados são apresentados ao usuário assim que terminada a configuração de uma nova missão, assim como quantidade de fotos a serem capturadas e tempo de voo para concluir a missão.

O aplicativo apresenta um mapa com dois botões (Figura 2, canto inferior direito), sendo utilizados para centralizar o mapa com a posição atual do Drone e para iniciar a configuração de uma nova missão. Na parte superior da tela, informações como modo de

\footnotetext{
${ }^{1}$ Waypoints são pontos no globo terrestre precisamente definido por coordenadas geográficas através do sistema GPS.
} 
voo, altura, velocidades horizontal e vertical, além de nível de baterias são apresentadas ao usuário e atualizadas em tempo real.

Criando uma nova missão, instruções são apresentadas ao usuário indicando como deverão ser inseridos os waypoints no mapa (Figura 2 B). No mapa também é apresentada a localização do Drone quando parado, ou em movimento (Figura 2 C). Após inseridos os waypoints e abrindo a configuração de missão (Figura 2 D), o usuário tem a opção de selecionar a altura que será efetuado o monitoramento, e todos os cálculos são feitos de forma automática, mostrando somente informações como distancia à percorrer e número de imagens a serem capturadas. Ao fim, quando acionado o botão FINISH, os waypoints são enviados ao Drone, liberando um botão de inicio de missão, que quando iniciada, transforma-se em botão de cancelar missão ou vice-versa.
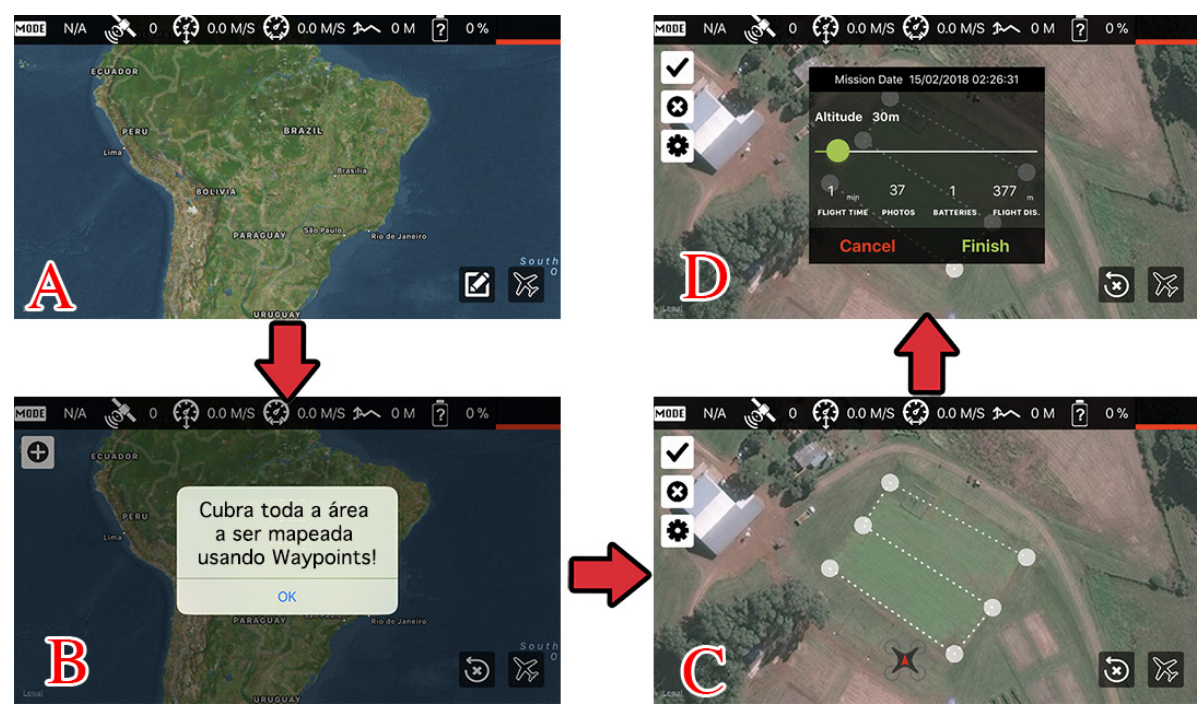

Figura 2. Tela inicial do aplicativo Autocapture (A), Instruções para a inserção de waypoints no mapa (B), waypoints marcados e pronto para configurar (C), configuração da missão, e informações sobre ela (D).

\section{Caso de uso do Aplicativo Auto Capture}

O aplicativo Auto Capture foi utilizado para o mapeamento de um campo experimental de trigo situado na cidade de Coxilha-RS, pertencente a EMBRAPA TRIGO (Lat: 28.186418 Long: -52.325192), durante o período de 10/08/2017 a 01/11/2017. Foram efetuados 6 mapeamentos da área utilizando os Drones DJI Phantom 3 PRO e DIY with DJI NAZA V2-GroundStation, os quais suportam a utilização do SDK. Cada coleta teve uma duração de 30 segundos, a uma altura de 30 metros para todas as capturas. Visando obter imagens com uma boa iluminação e consistência, as coletas foram efetuadas sempre no mesmo horário (12:00 PM UTC/GMT -3) [Cerbaro 2016].

Cada coleta resultou num total de 8 imagens, com um overlapping médio de 70\%, possibilitando a criação de mapa em alta resolução para o monitoramento da área. $\mathrm{Na}$ Figura 3 A, pode-se observar o mosaico RGB gerado por um software desenvolvido como parte complementar a este trabalho, assim como a Figura $3 \mathrm{~B}$, que por sua vez é um mosaico de imagens NDVI (Normalized Difference Vegetation Index) [Jose et al. 2014]. Estas coletas foram efetuadas no dia 21/09/2017. 

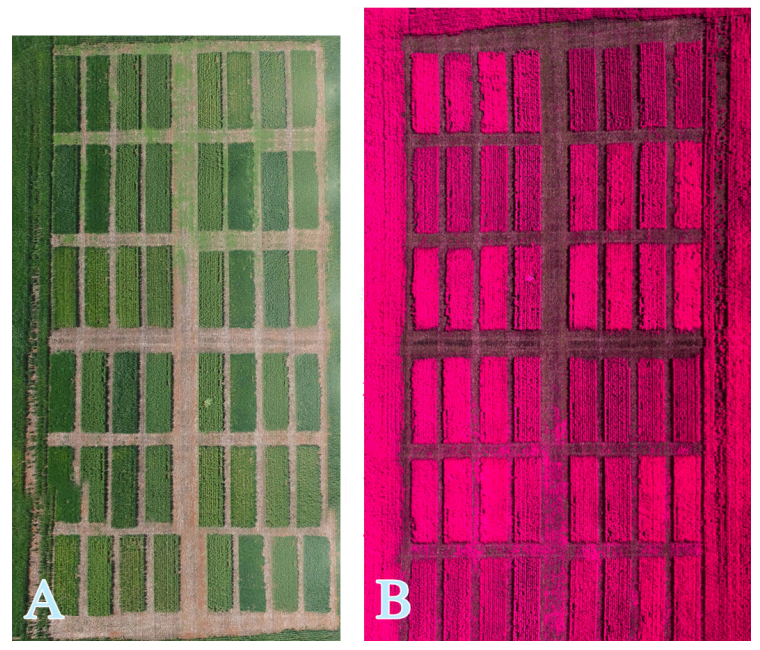

Figura 3. Mosaico RGB gerado por imagens capturadas por meio do Drone Phantom 3 PRO (A), Mosaico NDVI, capturadas por meio do Drone DIY Naza V2 (B).

\section{Conclusões}

Com as coletas realizadas na área da EMBRAPA TRIGO, o aplicativo mostrou-se capaz de capturar imagens de forma automática e simples, facilitando o trabalho de mapeamento do meio agrícola. Na parte funcional, onde se encaixa a exatidão no ponto de coleta de cada imagem, foram obtidos bons resultados, sendo que cada imagem teve uma média próxima a desejada de $70 \%$ de overlapping com sua vizinha.

\section{Referências}

Artioli, F. and Beloni, T. (2016). Diagnóstico do perfil do usuário de Drones no Agronegócio Brasileiro. Revista iPecege, 2(3):40-56.

Cerbaro, V. A. (2016). CROP DRONE : UMA SOLUÇÃO PARA COLETA, ARMAZENAMENTO E DISPONIBILIZAÇÃO DE DADOS. 1:93.

DJI-Developer (2018). Disponível em: http://developer.dji.com.

DJI-GS-PRO (2018). Disponível em: https://www.dji.com/ground-station-pro.

Ferreira, A., Roig, H., Marotta, G., and Meneses, P. (2013). Utilização de aeronaves remotamente pilotadas para extração de mosaico georreferenciado multiespectral e modelo digital de elevação de altíssima resolução espacial. Simposio Brasileiro de Sensoriamento Remoto, 1:9308-9315.

Henrique, J., Andreis, D., Pavan, W., Dalbosco, J., Delponte, E., Godoy, C. V., Mauricio, J., and Fernandes, C. Um Aplicativo iPhone para a Rastreabilidade da Ferrugem Asiática da Soja no Brasil.

Jose, B., Nicolas, M., Danilo, C., and Eduardo, A. (2014). Multispectral ndvi aerial image system for vegetation analysis by using a consumer camera. In Power, Electronics and Computing (ROPEC), 2014 IEEE International Autumn Meeting on, pages 1-6. IEEE. 